\title{
CONSTITUÍNTES FENÓLICOS POLARES DE Schinus terebinthifolius RADDI (ANACARDIACEAE)
}

Melina Ceruks, Paulete Romoff, Oriana A. Fávero e João Henrique G. Lago*

Departamento de Química, Centro de Ciências e Humanidades, Universidade Presbiteriana Mackenzie, Rua da Consolação, 930, 01302-907 São Paulo - SP, Brasil

Recebido em 24/3/06; aceito em 17/7/06; publicado na web em 10/1/07

POLAR PHENOLIC CONSTITUENTS FROM Schinus terebinthifolius Raddi (ANACARDIACEAE). The EtOH extract from the leaves of Schinus terebinthifolius showed anti-radicalar potential in the DPPH test. It was partitioned between $n$ - $\mathrm{BuOH}: \mathrm{H}_{2} \mathrm{O}(1: 1)$ and these two phases were also evaluated for anti-radicalar activity. The active $n$ - $\mathrm{BuOH}$ phase was partitioned between EtOAc: $\mathrm{H}_{2} \mathrm{O}$ (1:1) and the active EtOAc phase was submitted to chromatographic procedures to afford five active phenolic compounds: ethyl gallate, methyl gallate, quercitrin, myricetrin and myricetin. The structures of these compounds were established by NMR spectral data analysis.

Keywords: Schinus terebinthifolius; phenolic compounds; anti-radicalar potential.

\section{INTRODUÇÃOO}

Anacardiaceae é uma família botânica representada por 70 gêneros e cerca de 600 espécies de árvores ou arbustos, conhecidas por serem frutíferas e apresentarem madeira de boa qualidade ${ }^{1}$.

Schinus terebinthifolius Raddi, vernaculamente conhecida como aroeira-pimenteira, é uma árvore de porte médio, monóica, de folhas compostas e aromáticas sendo usada em culinária e, na França, recebe o nome de poivre rose, um tipo de pimenta doce. Ocorre desde o Rio Grande do Norte até o Rio Grande do Sul, em várias formações vegetais, sendo mais comum em beiras de rios ${ }^{2}$.

Estudos fitoquímicos e biológicos efetuados com espécies do gênero Schinus descrevem a ocorrência de terpenóides e ácidos graxos em $S$. molle $^{3-5}$ e em $S$. terebinthifolius ${ }^{6-10}$. Dentre os terpenóides, dois triterpenos isolados de $S$. terebinthifolius ${ }^{10}$ foram caracterizados como inibidores específicos da fosfolipase $\mathrm{A}_{2}$. A literatura cita ainda que o extrato em etanol das cascas do tronco de $S$. terebinthifolius e as frações em hexano, clorofórmio e em acetato de etila, provenientes da partição deste, foram ativos frente a Staphylococcus aureus ${ }^{11}$ e que os extratos aquosos de $S$. molle e de $S$. terebinthifolius inibiram o crescimento de Candida albicans ${ }^{12}$. Além disso, extratos de S. terebinthifolius ${ }^{13}$ e de $S$. weinmannifolia ${ }^{13}$ apresentaram atividade anti-radicalar em ensaios de peroxidação lipídica e com DPPH.

Em vista dos estudos químicos com $S$. terebinthifolius terem sido direcionados aos componentes de baixa e média polaridade, associado ao fato de que nenhum estudo foi até então realizado com o objetivo de conhecer as substâncias responsáveis pela atividade anti-oxidante observada para esta espécie ${ }^{13}$ efetuou-se, no presente trabalho, o estudo fitoquímico da fase em acetato de etila, oriunda do extrato em etanol das folhas de S. terebinthifolius com potencial anti-radicalar através de ensaio em CCD/DPPH, o qual, após sucessivas etapas de purificação com Sephadex LH20 , conduziu ao isolamento de galato de etila (1), miricetrina (2), quercitrina (3), galato de metila (4) e miricetina (5), descritas pela primeira vez neste gênero.

*e-mail: joaolago@iq.usp.br<smiles>O=C(O)c1cc(O)c(O)c(O)c1</smiles>

$1 \mathrm{R}=\mathrm{Et}$

$4 \mathrm{R}=\mathrm{Me}$<smiles>[R]c1cc(-c2oc3cc(O)cc(O)c3c(=O)c2[R])cc(O)c1O</smiles>

$2 \mathrm{R}=$ Ramnose $; \mathrm{R}^{\prime}=\mathrm{OH}$

$3 \mathrm{R}=$ Ramnose $; \mathrm{R}^{\prime}=\mathrm{H}$

$5 \mathrm{R}=\mathrm{H} ; \mathrm{R}^{\prime}=\mathrm{OH}$
Figura 1. Substâncias isoladas das folhas de Schinus terebinthifolius

\section{RESULTADOS E DISCUSSÃO}

O fracionamento cromatográfico do extrato em etanol das folhas de $S$. terebenthifolius, guiado pelo ensaio para detecção do potencial anti-radicalar, resultou no isolamento de cinco compostos fenólicos (1-5) ativos, cujas estruturas foram identificadas com base na análise dos dados obtidos dos espectros de RMN.

Os compostos 1-2 foram identificados, respectivamente, como galatos de etila e de metila pela comparação dos dados de RMN com os descritos na literatura ${ }^{14,15}$. A possibilidade de $\mathbf{1}$ ser artefato, devido à extração ter sido realizada com $\mathrm{EtOH}$, foi descartada quando o material vegetal foi extraído com $\mathrm{MeOH}$. Após a realização do processo de partição descrito na parte experimental, foi registrado um espectro de $\mathrm{RMN}$ de ${ }^{1} \mathrm{H}$ da fase AcOEt, o qual mostrou os sinais referentes ao grupo etoxílico, além daqueles referentes ao grupo metoxílico, indicativo da presença de ambos metabólitos.

$\mathrm{O}$ espectro de RMN de ${ }^{1} \mathrm{H}$ do composto 5 (DMSO-d $\mathrm{d}_{6}$ ) mostrou dois dubletos em $\delta_{\mathrm{H}} 6,38(J=1,8 \mathrm{~Hz}, 1 \mathrm{H})$ e $6,17(J=1,8 \mathrm{~Hz}, 1 \mathrm{H})$, relativos a hidrogênios acoplando em relação meta que, associados ao singleto em $\delta_{\mathrm{H}} 7,21(2 \mathrm{H})$, sugeriram o esqueleto de um flavonol. $\mathrm{O}$ espectro de RMN de ${ }^{13} \mathrm{C}$ (DMSO- $\mathrm{d}_{6}$ ) mostrou treze sinais, dos quais seis referentes aos carbonos do anel A, quatro referentes ao anel B e mais três atribuídos aos carbonos do sistema $\alpha, \beta$-insaturado (anel C) de um flavonol. A comparação dos dados espectrais com os da miricetina ${ }^{16}$ comprovou a identidade estrutural de $\mathbf{5}$.

$\mathrm{O}$ espectro de RMN de ${ }^{1} \mathrm{H}$ (DMSO-d ( $_{6}$ ) de 2 mostrou o mesmo perfil daquele observado para $\mathbf{5}$, exceto pela presença dos dubletos 
em $\delta_{\mathrm{H}} 5,18(J=1,0 \mathrm{~Hz}, 1 \mathrm{H})$ e em $\delta_{\mathrm{H}} 0,79(J=5,3 \mathrm{~Hz}, 3 \mathrm{H})$ e do multipleto entre $\delta_{\mathrm{H}} 3,1-4,0$, relativos a uma unidade glicosídica. No espectro de $\mathrm{RMN}$ de ${ }^{13} \mathrm{C}$ (metanol- $\mathrm{d}_{4}$ ) foram observados treze sinais relativos à aglicona além de seis referentes à ramnose, possibilitando, após comparação com dados da literatura ${ }^{16}$, a identificação de 2 como a miricetrina (miricetina-3-O- $\alpha$-ramnosídeo). Os espectros de RMN de ${ }^{1} \mathrm{H}$ e de ${ }^{13} \mathrm{C}$ de $\mathbf{3}$ mostraram-se muito similares aos de 2, inclusive em relação aos sinais referentes à unidade ramnosídica. No entanto, foram observados sinais adicionais em $\delta_{\mathrm{H}}$ $7,21$ (d, $J=1,7 \mathrm{~Hz}, 2 \mathrm{H}), 7,20$ (sl, 1H) e 6,86 (dd, $J=8,2$ e 1,7 Hz, $1 \mathrm{H})$, os quais são indicativos de um sistema 1,3,4-trissubstituído no anel B do flavonol. Após comparação dos dados de RMN descritos na literatura ${ }^{16,17}$ foi possível identificar a estrutura de $\mathbf{3}$ como a quercitrina (quercetina-3-O- $\alpha$-ramnosídeo). As configurações alfa para as unidades ramnosídicas das substâncias $\mathbf{2}$ e $\mathbf{3}$ foram definidas com base no valor da constante de acoplamento $(J=1,0 \mathrm{~Hz})$ dos dubletos relativos a H-1", o qual sugeriu um acoplamento di-equatorial entre $\mathrm{H}-1$ " e $\mathrm{H}-2$ "'18.

Todos os compostos isolados da fase ativa (1-5) foram submetidos à avaliação do potencial anti-radicalar, aspergindo-se uma solução de 2,0 mg/mL de DPPH em metanol em uma placa de CCD $\left(\mathrm{SiO}_{2}\right)$. Após $5 \mathrm{~min}$, foram observadas manchas amareladas sob fundo violeta, sugerindo que 1-5 são os responsáveis pelo potencial antiradicalar observado nos extratos testados.

A atividade anti-radicalar de flavonóides, ésteres do ácido gálico e aldeído caféico extraídos das folhas de Tachigalia paniculata (Leguminosae) foi avaliada usando-se três ensaios distintos ${ }^{19}$. Os autores observaram que dois derivados glicosídicos da miricetina e o galato de metila se mostraram ativos frente ao cátion radical ABTS; além disso, os dois derivados glicosídicos da miricetina foram as substâncias mais ativas no teste da xantina oxidase. Em outro trabalho ${ }^{20}$, os autores compararam as atividades do ácido gálico e de uma série de seus ésteres (galatos de metila, de propila, de laurila e de estearila) frente ao DPPH. Todas as substâncias foram mais ativas que o $\alpha$ tocoferol, indicando que, neste ensaio, o tamanho da cadeia alquílica não interferiu com a atividade observada. Assim, visto que ésteres do ácido gálico e flavonóides derivados da miricetina e quercetina apresentam elevado potencial anti-radicalar ${ }^{14,19-22}$, sugerimos que as substâncias isoladas no presente trabalho sejam as responsáveis pela atividade observada nos extratos avaliados.

\section{PARTE EXPERIMENTAL}

\section{Procedimentos gerais}

Os espectros de Ressonância Magnética Nuclear foram registrados em espectrômetro Brüker DPX-300 $\left({ }^{1} \mathrm{H}: 300 \mathrm{MHz}\right.$ e ${ }^{13} \mathrm{C}$ : $75 \mathrm{MHz}$ ) utilizando DMSO- $\mathrm{d}_{6}$ e metanol- $\mathrm{d}_{4}$ como solventes e como referência interna o sinal residual do solvente. Nas separações cromatográficas em coluna aberta usou-se Sephadex LH-20 (Aldrich); nas análises com camada fina foi utilizado gel de sílica $60 \mathrm{PF}_{254}$ (Merck) $(0,25 \mathrm{~mm}$ ) seguido de revelação com luz UV (254 e $366 \mathrm{~nm}$ ), além de solução metanólica $2,0 \mathrm{mg} / \mathrm{mL}$ de DPPH (radical 2,2-difenil-1-picril-hidrazil) (Sigma-Aldrich).

\section{Ensaio para detecção do potencial anti-radicalar}

As amostras foram aplicadas em placas de CCD, eluídas com sistemas de solventes adequados e aspergidas com uma solução 2,0 $\mathrm{mg} / \mathrm{mL}$ de DPPH em metanol. Após evaporação do solvente (cerca de $5 \mathrm{~min}$ ), o potencial anti-radicalar foi verificado através do aparecimento de manchas amarelas sob fundo violeta, de acordo com o descrito na literatura ${ }^{23}$.

\section{Material vegetal}

Folhas de Schinus terebinthifolius foram coletadas em Novembro de 2005 no Campus São Paulo da Universidade Presbiteriana Mackenzie (São Paulo-SP, Brasil). Uma exsicata (SP272591) encontra-se catalogada no Herbário do Instituto de Botânica de São Paulo - SP, Brasil.

\section{Extração e isolamento dos constituintes químicos}

Após secagem e moagem, $158 \mathrm{~g}$ das folhas de S. terebinthifolius foram submetidas à extração através de maceração com EtOH à temperatura ambiente. Após eliminação do solvente a vácuo obtiveramse 40,0 g de um material viscoso, de coloração esverdeada. Este extrato foi dissolvido em $n$-BuOH- $\mathrm{H}_{2} \mathrm{O}(1: 1)$ e ambas as fases obtidas foram testadas para detecção de atividade anti-radicalar em experimento de CCD/DPPH. Verificou-se nesta análise que os compostos ativos estavam concentrados na fase $n-\mathrm{BuOH}$. Após evaporação do solvente, acrescentou-se a esta fase solução de AcOEt: $\mathrm{H}_{2} \mathrm{O}$ (1:1), sendo avaliado o potencial anti-radicalar das duas fases obtidas. A fase em AcOEt $(9,8 \mathrm{~g})$ ativa foi submetida à cromatografia de exclusão (Sephadex LH-20 com MeOH), resultando em 34 frações de $15 \mathrm{~mL}$ cada. Após análise por CCD, estas foram reunidas em 6 grupos (I-VI) e novamente cada grupo foi submetido ao ensaio para detecção do potencial anti-radicalar, onde se observou que a atividade estava concentrada nos grupos II-VI. As frações reunidas do grupo II (frações 911) forneceram o galato de etila $(\mathbf{1}, 243 \mathrm{mg})$; enquanto que o grupo III (frações 12-20, $563 \mathrm{mg}$ ) foi novamente fracionado em Sephadex LH20 (metanol) para fornecer 34 frações (6 mL cada) que, após análise por CCD, foram reunidas em 4 grupos (III-1/III-4). Os grupos III-2 (frações 3-8), III-3 (frações 9-17) e III-4 (frações 24-32) forneceram, respectivamente, quantidade adicional de galato de etila $(\mathbf{1}, 236 \mathrm{mg})$, miricetrina $(\mathbf{2}, 172 \mathrm{mg})$ e quercitrina $(\mathbf{3}, 112 \mathrm{mg})$. As frações reunidas do grupo IV (frações 21-23, $638 \mathrm{mg}$ ) forneceram uma mistura constituida de galato de etila (1) e de galato de metila (4) na proporção 1:3, conforme análise por RMN de ${ }^{1} \mathrm{H}$. O material das frações reunidas do grupo V (frações 24-27, $289 \mathrm{mg}$ ) mostrou-se constituído somente pela miricetina (5). O grupo VI (frações 28-34) forneceu galato de metila $(\mathbf{4}, 378 \mathrm{mg})$.

Adicionalmente, uma pequena quantidade das folhas secas e moídas (10 g) foi extraída com $\mathrm{MeOH}$ e submetida aos processos de partição, descritos anteriormente. Da fase AcOEt obtida registrou-se um espectro de RMN de ${ }^{1} \mathrm{H}$ (DMSO- $\mathrm{d}_{6}$ ) com o objetivo de descartar a formação de $\mathbf{1}$ como artefato.

Galato de etila (1): RMN ${ }^{1} \mathrm{H}\left(300 \mathrm{MHz}, \mathrm{DMSO}_{6}\right) \delta_{\mathrm{H}}$ (mult; $J$ em Hz; H): 6,92 ( $s$; H-2/H-6), 4,18 ( $q$; 7,2; H-8), 1,25 ( $t ; 7,2 ; \mathrm{H}-9)$. RMN ${ }^{13} \mathrm{C}\left(75 \mathrm{MHz}, \mathrm{DMSO}-\mathrm{d}_{6}\right) \delta_{\mathrm{C}}: 119,6(\mathrm{C}-1), 108,5$ (C-2/C-6), 145,6 (C-3/C-5), 138,6 (C-4), 165,9 (C-7), 60,0 (C-8), 14,3 (C-9).

Miricetrina (2): RMN ${ }^{1} \mathrm{H}\left(300 \mathrm{MHz}, \mathrm{DMSO}-\mathrm{d}_{6}\right) \delta_{\mathrm{H}}$ (mult; $J$ em Hz; H): 7,04 ( $s$, H-2' e H-6'), 6,36 (d; 1,8; H-6), 6,18 (d; 1,8, H-8), 5,18 ( $d$; 1,0; H-1"), 3,1 - 4,0 (m; H-2" - H-5"), 0,79 (d; 5,3; H-6"). $\mathrm{RMN}^{13} \mathrm{C}(75 \mathrm{MHz}$, metanol-d $), \delta_{\mathrm{C}}: 158,3$ (C-2), 134,5 (C-3), 177,9 (C-4), 162,9 (C-5), 99,9 (C-6), 165,4 (C-7), 94,3 (C-8), 157,5 (C9), 105,0 (C-10), 122,5 (C-1'), 109,5 (C-2'), 146,4 (C-3'), 137,1 (C-4'), 146,4 (C-5'), 109,5 (C-6'), 102,0 (C-1"), 70,3 (C-2”), 70,4 (C-3"), 71,3 (C-4”), 69,6 (C-5"), 17,3 (C-6”).

Quercitrina (3): RMN ${ }^{1} \mathrm{H}\left(300 \mathrm{MHz}, \mathrm{DMSO}-\mathrm{d}_{6}\right) \delta_{\mathrm{H}}$ (mult; $J$ em $\mathrm{Hz} ; \mathrm{H}): 6,38$ ( $d$; 1,9; H-6), 6,19 (d; 1,9; H-8), 7,21 (d; 1,7; H-2'), 6,86 ( $d d ; 8,2$ e 1,7; H-5'), 7,20 (sl; H-6'), 5,23 ( $d ; 1,0 ; \mathrm{H}-1$ '), 3,1 3,9 ( $m$; H-2" - H-5"), 0,79 (d; 5,2, H-6") RMN ${ }^{13} \mathrm{C}$ (75 MHz, DMSO$\left.\mathrm{d}_{6}\right), \delta_{\mathrm{C}}: 156,9$ (C-2), 134,6 (C-3), 178,1 (C-4), 161,7 (C-5), 99, 2 (C6), 164,7 (C-7), 94,1 (C-8), 157,8 (C-9), 104,5 (C-10), 121,1 (C1'), 115,9 (C-2'), 145,6 (C-3'), 148,9 (C-4'), 116,0 (C-5'), 121,6 
(C-6’), 102,2 (C-1”), 70,5 (C-2”), 70,8 (C-3”), 71,6 (C-4”), 71,0 (C-5"), 17,7 (C-6").

Galato de metila (4): RMN ${ }^{1} \mathrm{H}\left(300 \mathrm{MHz}, \mathrm{DMSO}-\mathrm{d}_{6}\right) \delta_{\mathrm{H}}$ (mult; $J$ em Hz; H): 6,92 ( $s$; H-2/H-6), 3,73 ( $s ; \mathrm{H}-8) . \mathrm{RMN}{ }^{13} \mathrm{C}(75 \mathrm{MHz}$, DMSO-d $), \delta_{\mathrm{C}}: 119,4$ (C-1), 108,6 (C-2/C-6), 145,6 (C-3/C-5), 138,4 (C-4), 166,4 (C-7), 51,7 (C-8).

Miricetina (5): $\mathrm{RMN}{ }^{1} \mathrm{H}\left(300 \mathrm{MHz}, \mathrm{DMSO}-\mathrm{d}_{6}\right) \delta_{\mathrm{H}}\left(\right.$ mult; $^{\mathrm{e}} \mathrm{em}$ Hz; H): 7,21 ( $s$, H-2' e H-6'), 6,38 (d; 1,8; H-6), 6,17 (d; 1,8; H-8). $\mathrm{RMN}{ }^{13} \mathrm{C}\left(75 \mathrm{MHz}, \mathrm{DMSO}-\mathrm{d}_{6}\right), \delta_{\mathrm{C}}: 147,1$ (C-2), 136,1 (C-3), 176,1 (C-4), 161,0 (C-5), 98,5 (C-6), 164, 2 (C-7), 93,5 (C-8), 156,4 (C9), 103,3 (C-10), 121,3 (C-1'), 107,7 (C-2'), 146,0 (C-3'), 136,1 (C-4'), 146,0 (C-5'), 107,7 (C-6').

\section{AGRADECIMENTOS}

À FAPESP e ao MackPesquisa pelo apoio financeiro para o desenvolvimento desse trabalho.

\section{REFERÊNCIAS}

1. Pio Corrêa, M.; Dicionário de Plantas Úteis do Brasil e das Exóticas Cultivadas, Ministério da Agricultura-IBDF, Imprensa Nacional: Rio de Janeiro, 1984, vol. 1.

2. Lorenzi, H.; Árvores Brasileiras: manual de identificação e cultivo de plantas arbóreas nativas do Brasil. Ed. Plantarum: São Paulo, 1992, p. 8.

3. Pozzo-Balbi, T.; Nobile, L.; Scapini, G.; Cini, M.; Phytochemistry 1978, 17, 2107.

4. Terhune, S. J.; Hogg, J. W.; Lawrence, B. M.; Phytochemistry 1974, 13, 865.
5. Dominguez, X. A.; Carmona, J. F.; Venegas, R. B.; Phytochemistry 1971, 10, 1687.

6. Moneam, N. M. A.; Ghoneim, T.; J. Chromatogr., A 1986, 361, 391.

7. Lloyd, H. A.; Jaouni, T. M.; Evans, S. L.; Morton, J. F.; Phytochemistry 1977, 16, 1301.

8. Campello, J. P.; Marsaioli, A. J.; Phytochemistry 1975, 14, 2300

9. Campello, J. P.; Marsaioli, A. J.; Phytochemistry 1974, 13, 659.

10. Jain, M. K.; Yu, B. Z.; Rogers, J. M.; Smith, A. E.; Boger, E. T. A.; Ostrander, R. L.; Rheingold, A. L.; Phytochemistry 1995, 39, 537.

11. Lima, M. R. F.; Luna, J. D. S.; Santos, A. F.; Andrade, M. C. C.; Sant'Ana, A. E. G.; Genet, J. P.; Marquez, B.; Neuville, L.; Moreaun, N.; J. Ethnopharmacol. 2006, 105, 137

12. Schmourlo, G.; Mendonça-Filho, R. R.; Alviano, C. S.; Costa, S. S.; J. Ethnopharmacol. 2005, 96, 563.

13. Velázquez, E.; Tournier, H. A.; Buschiazzo, P. M.; Saavedra, G.; Schinella, G. R.; Fitoterapia 2003, 74, 91

14. The Merck Index; $9^{\text {th }}$ ed. Rahway, NJ, USA: Merck and Co. Inc., 1976.

15. Ma, X.; Wu, L.; Ito, Y.; Tian, W.; J. Chromatogr., A 2005, 1076, 212.

16. Agrawal, P. K.; Bansal, M. C.; Porter, L. J.; Foo, L. Y. Em Carbon-13 NMR of Flavonoids - Studies in Organic Chemistry 39; Agrawal, P. K., ed.; Elsevier Science Publishers B.V.: Amsterdam, 1989, vol. 39.

17. Markham, K. R.; Ternai, B.; Stanley, R.; Geiger, H.; Mabry, T. J.; Tetrahedron 1978, 34, 1389 .

18. Nasr, C.; Berrurier, M. H.; Guth, A. L.; Anton, R.; Phytochemistry 1986, 25 , 770 .

19. Cioffi, G.; D’Auria, M.; Braca, A.; Mendez, J.; Castillo, A.; Morelli, I.; De Simone, F.; De Tommasi, N.; J. Nat. Prod. 2002, 65, 1526.

20. Kikuzaki, H.; Hisamoto, M.; Hirose, K.; Akiyama, K.; Taniguchi, H.; J. Agric. Food Chem. 2002, 50, 2161.

21. Luo, X. D.; Basile, M. J.; Kennelly, E. J.; J. Agric. Food Chem. 2002, 50, 1379.

22. Aaby, K.; Hvattum, E.; Skrede, G.; J. Agric. Food Chem. 2004, 52, 4595

23. Cavin, A.; Potterat, O.; Wolfender, J. L.; Hostettmann, K.; Dyatmyko, W.; J. Nat. Prod. 1998, 61, 1497. 